\title{
HARDENING BY ANNEALING AND IMPLEMENTATION OF HIGH DUCTILITY OF ULTRA-FINE GRAINED ALUMINUM: EXPERIMENT AND THEORY
}

\author{
T. S. Orlova', N. V. Skiba ${ }^{2,3,4}$ A. M. Mavlyutov ${ }^{5}$, M. Yu. Murashkin ${ }^{3,6}$, R. Z. Valievi,6 \\ and M. Yu. Gutkin ${ }^{2,4,5}$
}

\author{
${ }^{1}$ Ioffe Institute, Russian Academy of Sciences, Polytekhnicheskaya 26, St. Petersburg 194021, Russia \\ ${ }^{2}$ Institute of Problems of Mechanical Engineering, Russian Academy of Sciences, Bolshoj 61, Vasil. Ostrov, \\ St. Petersburg 199178, Russia \\ ${ }^{3}$ St. Petersburg State University, Universitetskaya nab. 7/9, St. Petersburg 199034, Russia \\ ${ }^{4}$ Peter the Great St. Petersburg Polytechnic University, Polytekhnicheskaya 29, St. Petersburg 195251, Russia \\ 5ITMO University, Kronverksky pr. 49, St. Petersburg 197101, Russia \\ ${ }^{6}$ Institute for Physics of Advanced Materials, Ufa State Aviation Technical University, K. Marksa 12, \\ Ufa 450000, Russia
}

Received: November 12, 2018

\begin{abstract}
The influence of low temperature annealing and subsequent deformation on microstructure, strength and ductility was investigated for the first time for high pressure torsion (HPT) processed commercially pure Al. Extremely high increases in the conventional yield stress (up to $50 \%$ ) and ultimate tensile strength (up to $30 \%$ ) were obtained by annealing of the ultrafine grained (UFG) samples in the range $90-200{ }^{\circ} \mathrm{C}$ for $1 \mathrm{~h}$. Such increases were accompanied by a sharp drop in ductility up to $1 \%$. Implementation of high ductility at the level of coarse-grained Al, while maintaining high strength of the HPT-processed sample was demonstrated for the first time and achieved by repeating the low temperature annealing followed by subsequent additional HPT deformation. The key role of relaxation of non-equilibrium high-angle grain boundaries (GBs) in the strengthening effect of UFG-Al by annealing is shown. Two theoretical models are suggested to explain the hardening by annealing and the implementation of high ductility in UFG structures. Within the models, plastic deformation occurs through emission of lattice dislocations from triple junctions of GBs containing pile-ups of grain-boundary dislocations, glide of the lattice dislocations across neighboring grains, their accumulation at and climb along the opposite GBs. The energy characteristics and the critical stresses of dislocation emission are determined in two different cases, for UFG Al subjected to annealing only and to annealing with subsequent additional HPT deformation. The calculated theoretical dependences of the flow stress on the plastic deformation value well fit qualitatively and quantitatively to our experimental data.
\end{abstract}

\section{INTRODUCTION}

Ultrafine grained (UFG, $100 \mathrm{~nm}<$ grain size $d<1$ $\mu \mathrm{m})$ or nanocrystalline (NC, $d<100 \mathrm{~nm}$ ) metals fabricated by severe plastic deformation (SPD) processes have been extensively investigated due to their high strength compared to coarse-grained (CG) metals; see e.g., [1-11]. Much attention has been paid to the UFG/NC face-centered cubic (FCC) materials, which demonstrate unique mechanical behaviors such as high strength, reduced or even no strain hardening, high strain rate sensitivity, etc. [12-14]. These unique mechanical properties of UFG/NC FCC metals are explained by the underlying deformation mechanisms in which grain boundaries (GBs) play the key role [13-17]. Realization of plastic deformation in NC structures by a nucleation of dislocations from GBs, their propagation 
through the grain, and the absorption at GBs was demonstrated in MD simulations [15]. Yang et al. [13] proposed a model based on the absorption of dislocation pile-ups by GBs to explain work softening. The interaction of dislocations with GBs in UFG aluminum was directly observed in in situ TEM loading-unloading experiments [16]. Sauvage et al. [17] showed that GBs in UFG metals differ from regular GBs by atomic structure and stress fields. Stressstrain responses such as increased yield stress and yield point drop were analyzed for UFG FCC polycrystal from the viewpoint of grain size and dislocation density in FE simulations [18].

In addition to the above unique properties of UFG/ $\mathrm{NC}$ metals, recently it has been found that postprocess annealing and subsequent plastic deformation can strongly affect the mechanical behavior of nanostructured metals in unexpected way. In particular, Huang et al. [19] observed that nanostructured Al can be hardened by annealing at low temperatures and softened when subsequently deformed, which is in contrast to the typical behavior of ordinary CG metals. When commercial purity (CP) $(99.2 \%)$ UFG Al fabricated by accumulative roll bonding (ARB) process with the equivalent strain of 4.8 was annealed at a relatively low temperature of $150{ }^{\circ} \mathrm{C}$ for $0.5 \mathrm{~h}$, the yield stress increased by about $9 \%$ from 259 to $281 \mathrm{MPa}$. Such hardening was accompanied by a strong decrease in ductility. The phenomenon was named as "hardening by annealing". When subsequent cold rolling with $15 \%$ of rolling reduction was applied to the annealed UFG samples, the yield stress reduced and the elongation increased so that the stress-strain curve became similar to that before the low temperature annealing. This phenomenon was called as "softening by deformation".

Hardening by annealing of UFG AI was also observed in a number of works [20-24]. Hardening by annealing is not typical for $C G$ metals and supposes new mechanism(s) which can operate only in metals with UFG or nanocrystalline microstructure.

Huang et al. [19] attributed the origin of hardening by annealing and softening by deformation to the limitation and the increase of intragrain dislocation sources in UFG structure due to low-temperature annealing and the low degree of cold rolling, respectively. According to Horita et al. [1], the decrease in the number of sources of intragrain mobile dislocations occurs due to their sink into nearby high-angle grain boundaries (GBs) during annealing. Using TEM, the interior dislocation density was showed to decrease from $1.33 \cdot 10^{14} \mathrm{~m}^{-2}$ in the ARBdeformed state to $5.3 \cdot 10^{13} \mathrm{~m}^{-2}$ after annealing at 150
${ }^{\circ} \mathrm{C}$ for $0.5 \mathrm{~h}$. Later Miyajima et al. [25], using scanning transmission electron microscopy (STEM) observations in combination with precise measurements of electrical resistivity, obtained a comparable change in the density of interior dislocations from an initial value of $1.3 \cdot 10^{14} \mathrm{~m}^{-2}$ down to $4 \cdot 10^{13} \mathrm{~m}^{-2}$ for ARB-deformed CP Al (99.1\%) after a similar lowtemperature annealing. The authors [25] concluded that the change in dislocation density was not dramatically high to explain the origin of the abnormal mechanical properties of UFG metals. They supposed that hardening by annealing and softening by subsequent deformation were probably somehow related to the thermally activated depinning of dislocations emitted from GBs, as is the case in the theory proposed by Kato [26].

It should be noted that hardening by annealing was not observed in a number of UFG structures of commercially pure (CP) Al. For example, for CP Al (99.5 wt.\%) in which the UFG structure was obtained by rotary swaging [27] or equal channel angular pressing [28], the subsequent annealing at temperatures up to $175^{\circ} \mathrm{C}$ did not lead to any change in microhardness. The inconsistency of literature data on the influence of annealing on the aluminum strength is most likely due to specific features of the UFG structures obtained by various methods and regimes of SPD. Therefore, it seems important to investigate the effect of annealing and subsequent deformations on Al nanostructured by different methods of SPD, comparing the specific microstructural features with the resulting mechanical behavior.

In the present work, the influence of low temperature annealing in a temperature range of 90$400{ }^{\circ} \mathrm{C}$ and low degree additional deformation on microstructure and resulting strength characteristics (the conventional yield stress and ultimate tensile stress) and ductility have been investigated for the first time for high pressure torsion (HPT) processed CP Al. A dramatic increase in the conventional yield stress by annealing and a drastic increase (on the level typical for $\mathrm{CG} \mathrm{Al}$ ) in ductility by subsequent deformation have been obtained for UFG aluminum for the first time. We have tried to elucidate the key microstructural parameters responsible for hardening by annealing and for significant increase in ductility after a small additional plastic deformation. On the basis of the obtained experimental results, theoretical models explaining hardening by annealing and the implementation of high plasticity while maintaining a high level of strength have been proposed for UFG structures. To our best knowledge, no plastic deformation micromecha- 
nisms explaining hardening by annealing and softening by subsequent deformation have been suggested until now.

\section{MATERIAL AND EXPERIMENTAL PROCEDURES}

The CP aluminum A5E (min 99.5 wt.\% Al, further denoted as $\mathrm{Al}$; grade aluminum $\mathrm{A} 5 \mathrm{E}$ corresponds to the chemical composition of the grades of aluminum 1350, registered by the American aluminum Association) was chosen as the material for this study. In order to obtain UFG structure, cylindrical bars $9 \mathrm{~mm}$ in diameter and $8 \mathrm{~mm}$ in height were cut from an initial rod, compressed under a pressure of $6 \mathrm{GPa}$ to a height of $2 \mathrm{~mm}$ and subjected to SPD processing by HPT under a hydrostatic pressure of $6 \mathrm{GPa}$ to 10 revolutions $(n=10)$ at room temperature (RT). The HPT treatment resulted in $1 \mathrm{~mm}$-thick disks $20 \mathrm{~mm}$ in diameter. The resulting HPT true strain $\gamma$ was estimated using the following equation [29]:

$$
\gamma=\ln \frac{2 \pi n r}{h}+\ln \frac{h_{0}}{h}
$$

where $r$ is the distance from the center of the sample, $h_{0}$ and $h$ are the disk height before and after HPT, respectively. According to Eq. (1), after $n=10$ the true strain at $r=5 \mathrm{~mm}$ was $\gamma \approx 6.4$. The HPT-processed samples were annealed at various temperatures in the range $90-400{ }^{\circ} \mathrm{C}$ for $1 \mathrm{~h}$ to obtain a variety of microstructural parameters (grain size, GB misorientation angle, dislocation density, etc.). Hereafter, the HPT-processed samples without annealing are referred to as AI_RT, the samples with subsequent annealing at a temperature $T_{a n}$ are referred to as Al_ $T_{\text {an }}$ (for example Al_90, Al_130, and so on).

Microstructure of the obtained samples was studied by electron backscattering diffraction (EBSD) and X-ray diffraction (XRD) analysis. The EBSD studies were performed using the scanning electron microscope Zeiss Merlin with a scan step of 0.2 $\mu \mathrm{m}$. The diffraction patterns were indexed using seven Kikuchi bands. For each sample under study, an area containing at least 2000 grains was analyzed to determine the distributions of grains on size and GBs between the adjacent grains on their misorientation angle $(\theta)$. EBSD maps were taken from the plane parallel to the disk plane at a distance of $5 \mathrm{~mm}$ from the disk center. The area of each grain determined during the EBSD mapping was approximated by the area of a circle, the diameter of which was taken as a grain size [30]. The average grain size $\left(d_{a v}\right)$ was calculated from the obtained distributions of grains on size. GBs with misorientation angle $\theta \geq 15^{\circ}$ were referred to highangle GBs (HAGBs), the percentage of HAGBs is further denoted as $t_{\geq 15}$. XRD measurements were taken on the Bruker D8 DISCOVER diffractometer in the regime of symmetric $\varphi-2 \varphi$ scanning with a parallel radiation beam CuK $\alpha(40 \mathrm{kV}, 40 \mathrm{~mA})$. The scanning step was $\Delta 2 \varphi=0.02^{\circ}$ and exposition time was $0.5 \mathrm{~s}$. The Pauli analysis method using TOPAS 5.0 software was applied to determine lattice parameter (a), average size of coherent-scattering regions $\left(D_{X R D}\right)$ and elastic microdistortion level $\left(<\varepsilon^{2}>^{1 / 2}\right)$. The dislocation density $L_{d i s}$ was estimated from the data obtained as follows [31]:

$$
L_{d i s}=2 \sqrt{3} \frac{<\varepsilon^{2}>^{1 / 2}}{D_{X B D} b},
$$

where $b$ is the Burgers vector magnitude for a perfect lattice dislocation.

To perform mechanical tests, blade-shaped specimens with a gauge width of $2 \mathrm{~mm}$ and a gauge length of $6 \mathrm{~mm}$ were cut from HPT-processed disks using the electric-spark machine. The uniaxial tensile test was performed on a Shimadzu AG-XD Plus machine with a constant strain rate of $510^{-4} \mathrm{~s}^{-1}$. Sample straining was recorded using a TRViewX $55 S$ video extensometer. At least 3 samples were tested for each state. The conventional yield stress $\left(\sigma_{0.2}\right)$ corresponding to $0.2 \%$ strain, ultimate tensile strength $\left(\sigma_{\mathrm{UTS}}\right)$ and relative elongation to failure $(\delta)$ were determined from the stress-strain diagrams obtained.

\section{EXPERIMENTAL RESULTS AND DISCUSSION}

The mechanical properties of CPAl were measured by tensile tests at room temperature. Fig. 1 shows the typical tensile curves of CP Al in the initial CG state, after HPT processing and subsequent annealing at $T_{a n}=150^{\circ} \mathrm{C}$ and additional deformations. The variations in $\sigma_{\mathrm{UTS}}, \sigma_{0.2}$, and $\delta$ for all studied states are presented in the form of diagrams in Fig. 2. As is seen, after HPT processing, $\sigma_{0.2}$ and $\sigma_{\mathrm{UTS}}$ increased by a factor of about 4 times, but $\delta$ decreased more than twice. These results agree well with the data of $[19,21,22,27,32-34]$, in which substantial increase in strength characteristics and decrease in ductility were obtained for CP Al with UFG structures formed by other methods, such as accumulative roll bonding, rotary swaging, and equal-channel angular pressing. Subsequent annealing for $1 \mathrm{~h}$ at $T_{\text {an }}$ in a temperature range of $90-200^{\circ} \mathrm{C}$ 


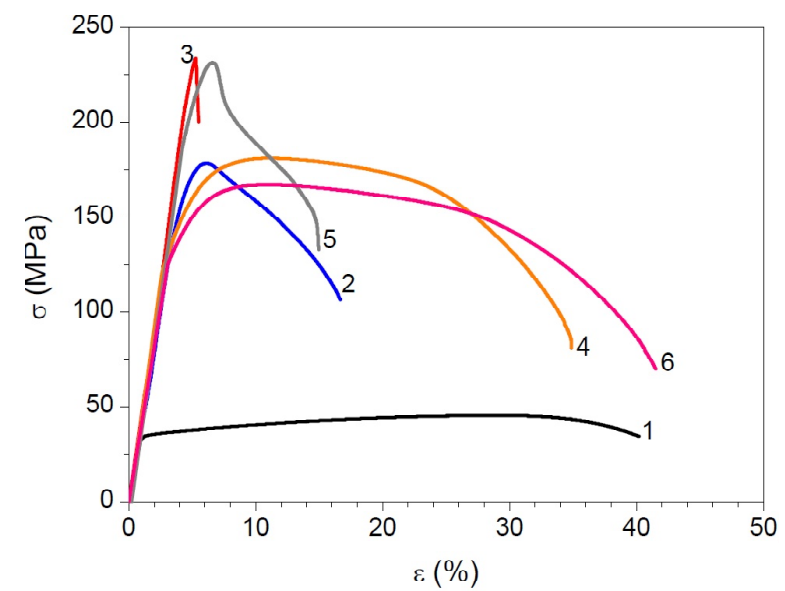

Fig. 1. Stress-strain diagram of CP Al specimens: (1) initial CG state, (2) after 10-revolution HPT at room temperature, (3) after HPT and annealing at $150^{\circ} \mathrm{C}$; (4) after the treatment similar to the treatment for (3) plus 0.25-revolution HPT at room temperature; (5) the same treatment as that for (4) plus annealing at $150^{\circ} \mathrm{C}$ for $1 \mathrm{~h}$; (6) the same treatment as that for (5) plus 0.25-revolution HPT at room temperature.

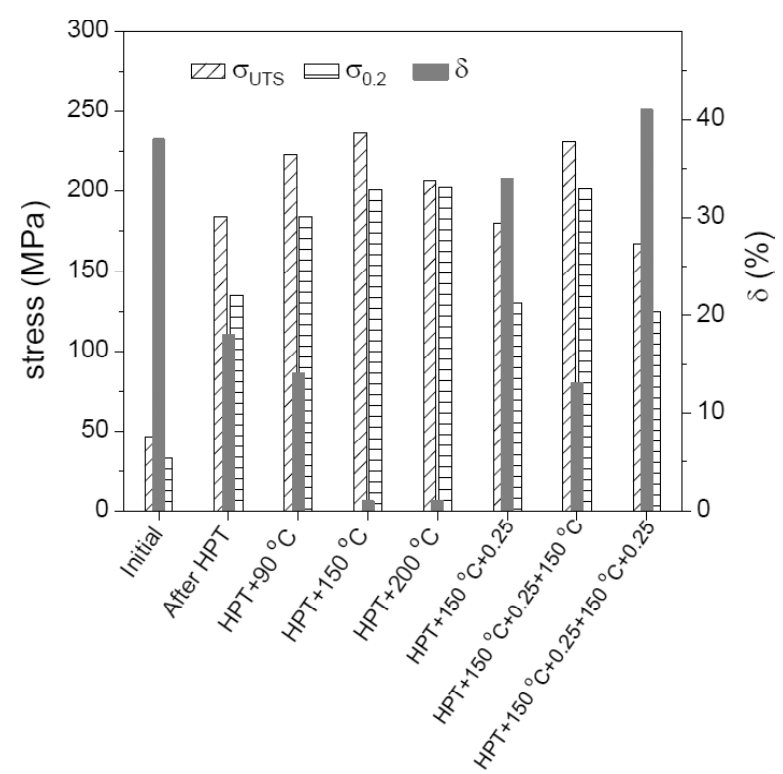

Fig. 2. Variation in ultimate tensile strength, conventional yield stress and ductility with annealing temperature and subsequent additional deformation for HPT-processed CPAI. The mechanical properties of CP Al in the initial CG state are also presented for comparison.

provided a further increase in $\sigma_{0.2}$ and $\sigma_{\text {UTS }}$ up to $50.4 \%$ and $28.8 \%$, respectively (Fig. 2 ), while $\delta$ dropped to $1 \%$. It should be noted that such strong hardening by annealing was achieved for the first time. Huang et al. [19] reported the increase in $\sigma_{0.2}$ by $8.9 \%$ after annealing at $150{ }^{\circ} \mathrm{C}$ for $0.5 \mathrm{~h}$. The comparison of the obtained values of annealing-induced effect for UFG CP Al processed by different methods of SPD is presented in Table 1.

In our work, the maximum increase in the strength accompanied by the minimum value $\delta \approx 1 \%$ was obtained for $T_{a n}=150^{\circ} \mathrm{C}$. This state (HPT +150 ${ }^{\circ} \mathrm{C}$ ) was chosen for study of possible effect of softening by subsequent deformation. The subsequent deformation (additional HPT deformation by 0.25 revolution corresponding to the additional true strain $\Delta \gamma \approx 0.012$ ) was applied after the annealing. As is seen in Figs. 1 and 2, such deformation substantially increased the ductility. Strength, although decreasing, but retained the values typical for the HPT state. It was found that repeating the procedure consisting of annealing at $150^{\circ} \mathrm{C}$ and subsequent deformation by 0.25 revolution leads to a record increase in ductility $(\delta \approx 42 \%)$ while maintaining strength at the level typical for the HPT state (Figs. 1 and 2). These findings point to a route for development of strong and ductile UFG materials based on (HPT) severe plastic deformation followed by twice repeating treatment consisting of an appropriate annealing and minor subsequent torsional deformation.

Detailed microstructural analysis was conducted to understand the underlying mechanisms responsible for the annealing-induced hardening. The microstructure was characterized by XRD and EBSD analyses. Fig. 3 presents typical EBSD maps of CP Al after HPT processing (a) and subsequent annealing at $150{ }^{\circ} \mathrm{C}(\mathrm{d})$, as well as the grain size distribution (b,e) and distribution of GBs on misorientations (c, f) obtained on the basis of these maps. Such characteristics were obtained for all the studied states. Fig. 4 shows the average grain size and fraction of HAGBs for these states. As is seen, the average grain size remains practically unchanged after annealing at $90^{\circ} \mathrm{C}$ and only slightly increases under annealing up to $200^{\circ} \mathrm{C}$.

The XRD patterns of CPAl in three states - after HPT processing, after subsequent annealing at 150 ${ }^{\circ} \mathrm{C}$, and after additional deformation by 0.25 revolution - are presented in Fig. 5 which demonstrates the peak narrowing after annealing and the peak broadening after subsequent additional deformation. The results of XRD analysis are shown in Table 2. Since annealing practically did not change the value of $D_{X R D}$, but resulted in pronounced decrease in the value of $\left\langle\varepsilon^{2}\right\rangle^{1 / 2}$, changes in the microdistorsion level can be referred to changes in the dislocation density. Indeed the line broadening analysis from Williamson and Smallman is not very accurate and 


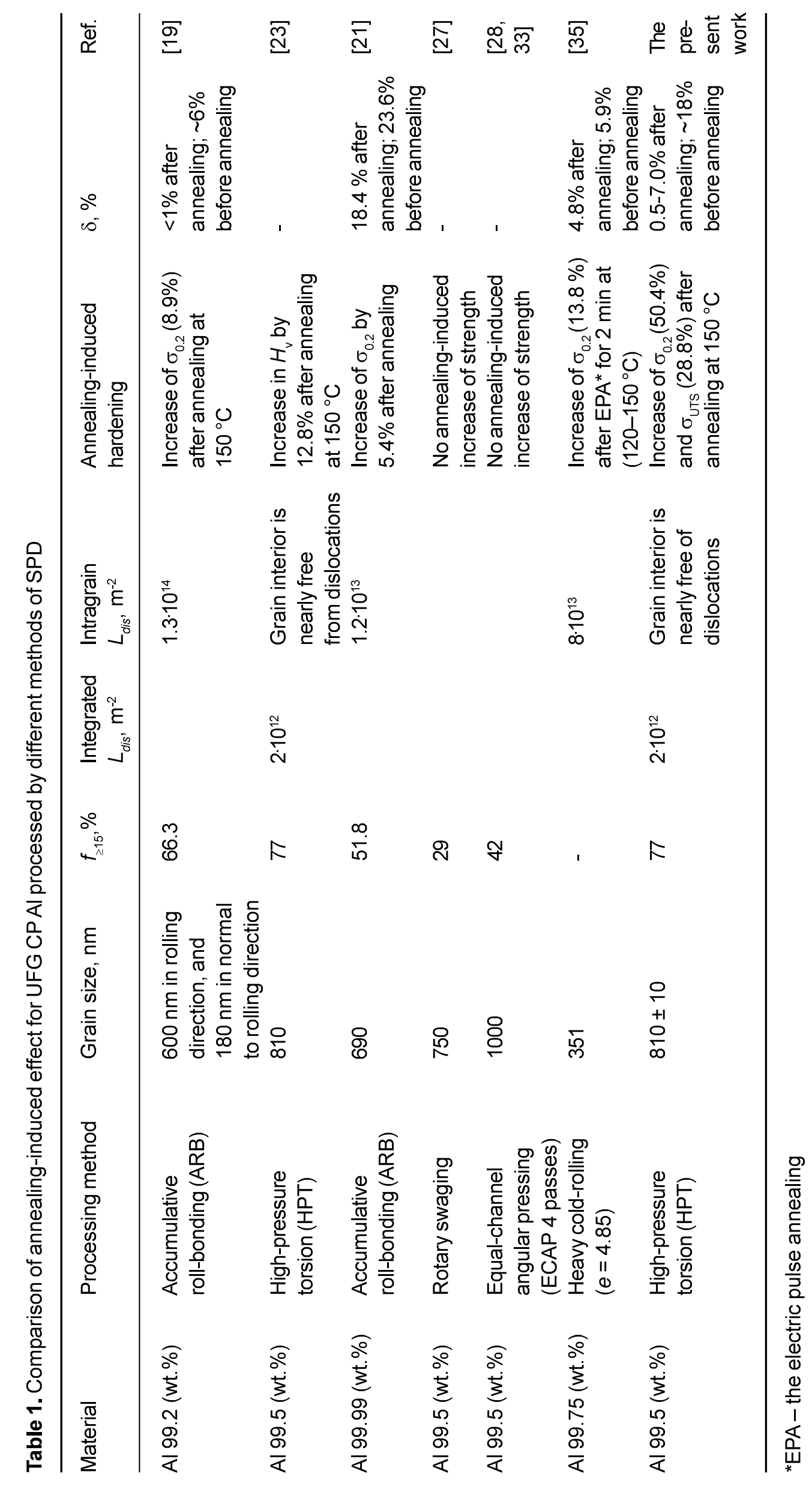



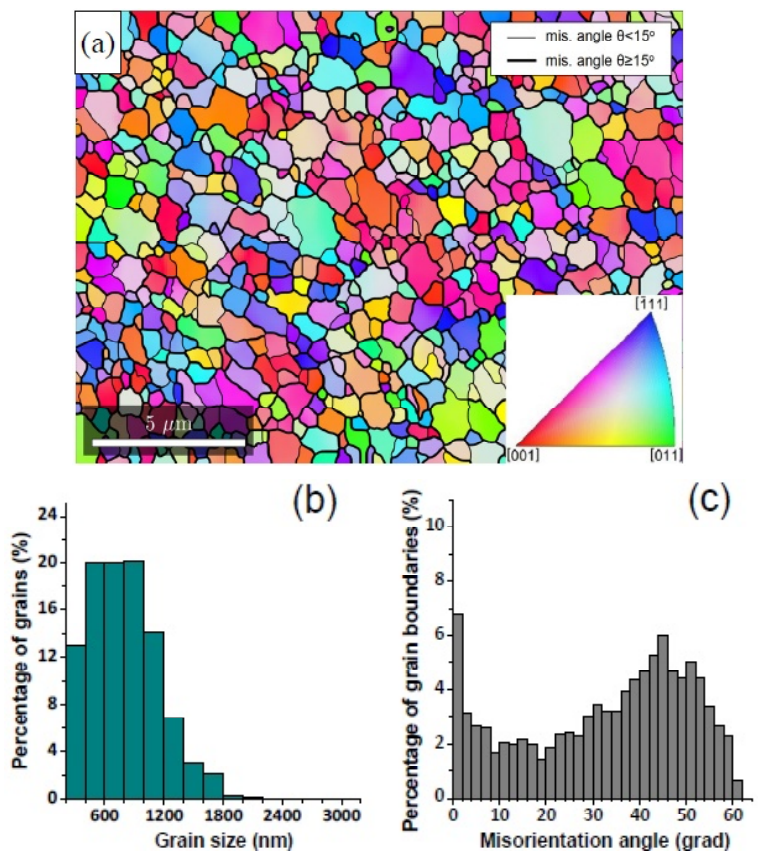
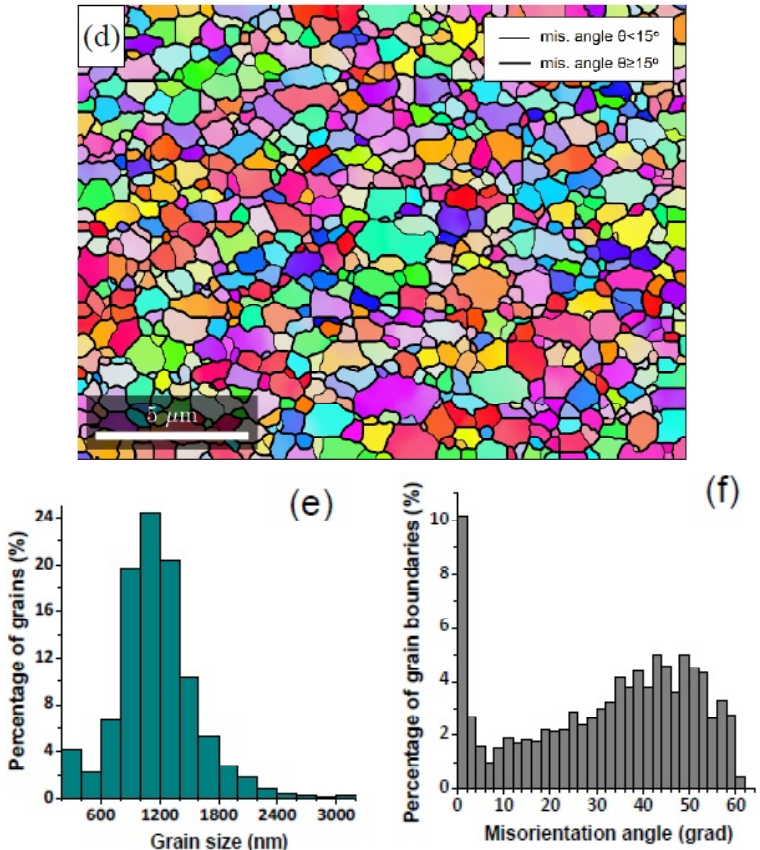

(f)

Fig. 3. EBSD maps and corresponding grain size distribution and distribution of grain boundaries on misorientation angle $\theta$ for HPT-processed CP Al before $(a, b, c)$ and after annealing at $150^{\circ} \mathrm{C}(\mathrm{d}, \mathrm{e}, \mathrm{f})$.

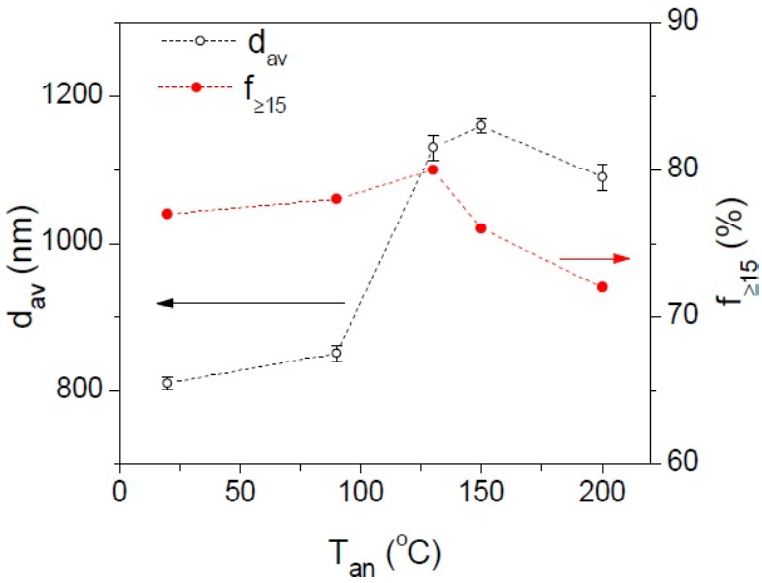

Fig. 4. Annealing temperature dependence of the grain size and the fraction of high-angle grain boundaries for HPT-processed CP Al.

does not give very accurate absolute values of dislocation densities. However, our comparative study of the influence of annealing and additional deformation on the XRD patterns in the same geometry shows a correct trend. We repeated the series of such measurements three times and confirmed the trend shown in the table. The dislocation density in the HPT-processed CP Al is not high and is equal to $\sim 4.1 \cdot 10^{12} \mathrm{~m}^{-2}$. The subsequent annealing at 150 ${ }^{\circ} \mathrm{C}$ leads to a significant decrease in the dislocation density (by a factor of almost 3 ). The subsequent additional small HPT deformation provides a substantial increase in the dislocation density nearly restoring the level characteristic of the unannealed UFG structure. Earlier we have showed by transmission electron microscopy that HAGBs in the HPT-processed CPAl demonstrate specific features of non-equilibrium character, namely, contours of extinction at GBs are extended in these samples and they become much narrower already after annealing at $90^{\circ} \mathrm{C}$ despite the grain size and portion of HAGBs do not change [36].

Moreover, only few dislocations were found within the grains by TEM in both HPT-processed samples

Table 2. Results of X-Ray diffraction analysis of CP Al specimens after HPT treatment, subsequent annealing at $150{ }^{\circ} \mathrm{C}$ and additional HPT by 0.25 turns. $D_{X R D}$ is the average size of coherent scattering regions, $\left\langle\varepsilon^{2}\right\rangle^{1 / 2}$ is the level of crystal lattice microdistorsions, $L_{d i s}$ is the dislocation density.

\begin{tabular}{llll}
\hline Specimen & $D_{X R D}, \mathrm{~nm}$ & $<\varepsilon^{2}>^{1 / 2}, \%$ & $L_{\text {dis }}, \mathrm{m}^{-2}$ \\
\hline Al_RT & $240 \pm 40$ & $0.008 \pm 0.003$ & $\sim 4.1 \cdot 10^{12}$ \\
Al_150 & $250 \pm 50$ & $0.003 \pm 0.001$ & $\sim 1.5 \cdot 10^{12}$ \\
Al_150+0.25 & $300 \pm 60$ & $0.010 \pm 0.003$ & $\sim 4.1 \cdot 10^{12}$ \\
\hline
\end{tabular}




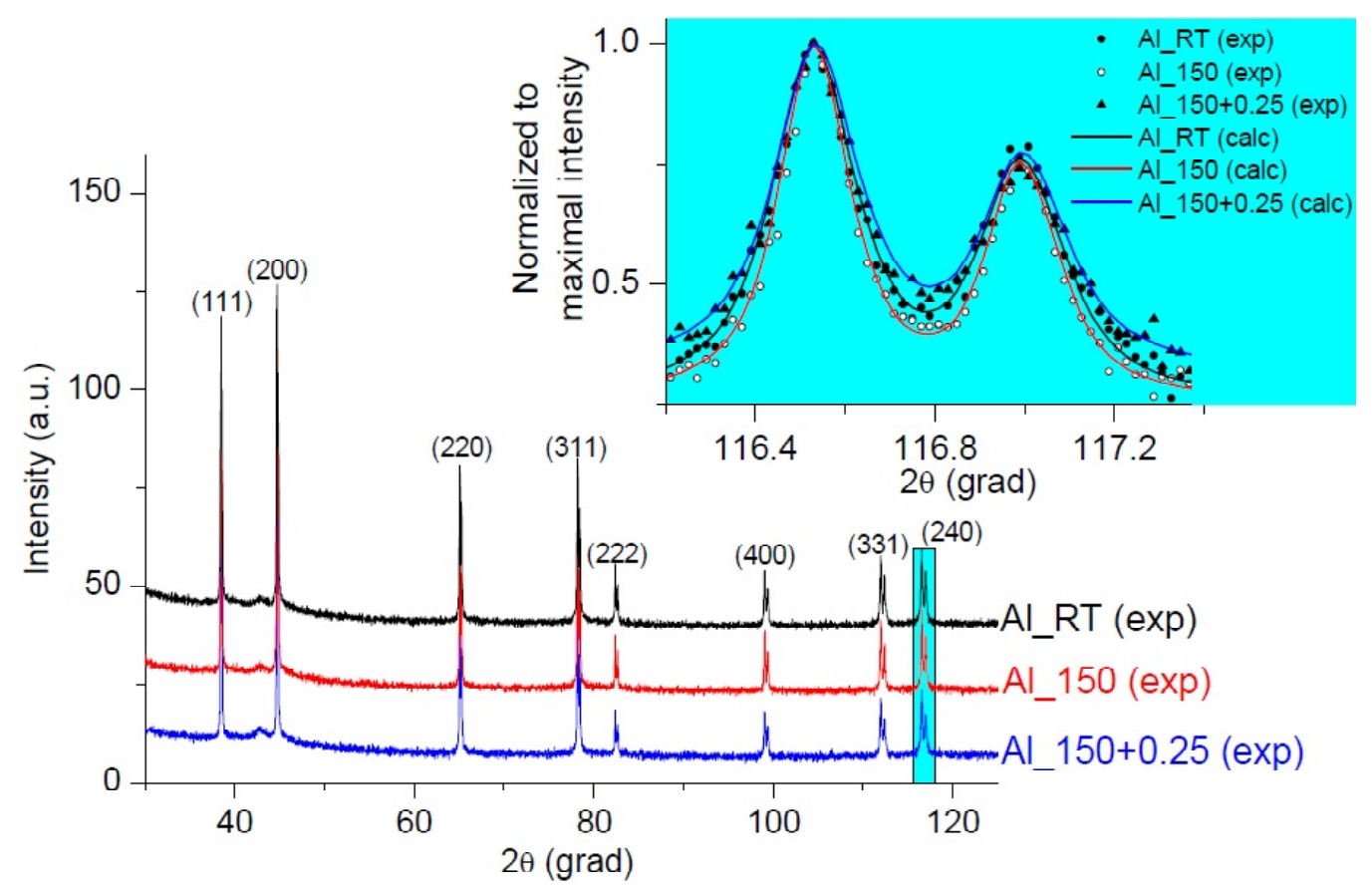

Fig. 5. The X-ray diffraction patterns of CP Al samples after high pressure torsion (AI_RT), after subsequent annealing (Al_150) and after additional small deformation (Al_150+0.25). The inset shows both the experimental results and simulation results for the parts of the XRD patterns denoted by the box.

before and after annealing at $90^{\circ} \mathrm{C}$ [36]. It means that dislocations mostly locate near GBs [36].

According to $[37,38]$, the extinction contour broadening near GBs in SPD-processed metals is caused by internal stresses and crystalline lattice distortions near GBs. In particular, Islamgaliev and Valiev [38] performed their analysis of extinction contours on TEM images of Cu structured by SPD. They obtained strain distribution in dependence on distance from a GB. Valiev et al. [2] showed that such strain distribution can be described as a strain field from an ensemble of GB dislocations (GBDs), more precisely, of extrinsic GBDs which are capable to glide within their GBs. For Al-0.5Mg alloy, structured by HPT, the presence of extrinsic dislocations in GBs was directly shown by high resolution electron microscopy [39]. Recently, based on a lattice image taken with an aberration-corrected transmission electron microscope for nanocrystalline $\mathrm{Pd}$, a comprehensive strain analysis has been performed and the results have been compared with a molecular dynamics simulation [40]. In particular, the authors showed that the strain field of the core of the triple junction has the characteristics of a dislocation.

Thus, we have demonstrated the existence of hardening by annealing in a temperature range of 90-200 ${ }^{\circ} \mathrm{C}$ for HPT-processed CP Al. It has been also found that it is possible to achieve high ductil- ity at the ductility level of CG Al while maintaining high strength of the HPT-processed sample by repeating the treatment consisting of low temperature annealing followed by a slight additional deformation by HPT. The UFG structure of the HPT-processed samples had the following specific microstructure features: (1) most of GBs belonged to HAGBs which demonstrated specific features of non-equilibrium character; (2) the initial dislocation density was rather low $\left(4.1 \cdot 10^{12} \mathrm{~m}^{-2}\right)$ and mostly attributed to GBs [36]; (3) the grain interior was nearly free of dislocations [36]. The additional small deformation of the annealed samples nearly restored the dislocation density to the value which is typical for the HPT-processed samples before annealing.

A comparison of the obtained results with the literature data is given in Table 1. It can be seen that the effect of hardening by annealing takes place only for those UFG structures in which the majority of GBs are HAGBs, while does not depend on the initial dislocation density in grain interior, i. e. the annealing-induced hardening takes place in both the UFG structure with high intragrain dislocation density and UFG structure with nearly dislocation-free grain interior. Definitely, the presence of dominant amount of HAGBs seems to be a necessary requirement to observe the annealing-induced effect. However, our findings show that this effect appeared after relaxation of HAGBs during annealing. Me- 
chanical testing of the UFG structure with the relaxed structure of HAGBs shows an increase of strength and sharp decrease of ductility. Such relaxation of GB structure can be due to rearrangement of dislocations inside GBs that can be accompanied by annihilation of GBDs without accepting intragrain mobile dislocations as in our case or with accepting mobile intragrain dislocations as in the case studied by Huang et al. [19]. It is important to note that low temperature annealing did not practically change the portion of HAGBs that supports our suggestion that the key microstructural parameter of UFG structure to demonstrate the annealing-induced hardening is the presence of dominant amount of non-equilibrium HAGBs, the energy of which could be decreased by appropriate annealing. Our results and conclusions are in good agreement with the results of Hasnaoui et al. [41]. Using molecular dynamic simulations of nanocrystalline $\mathrm{Ni}$, the authors showed that short-term annealing leads to relaxation of non-equilibrium GBs and such relaxation results in reduction of plasticity (increase of strength). Hu et al. [42] demonstrated a colossal increase of microhardness by annealing for nanograined $\mathrm{Ni}$ and $(\mathrm{Ni}-\mathrm{Mo}$ ) alloys, and showed experimentally that it occurred as a result of relaxation of GBs and grain boundary segregation of Mo.

Since the CP Al is not completely pure metal, but contains some amount of impurities, it is important to consider their influence on the observed effects of hardening by annealing and softening by subsequent deformation. No formation of any secondary phases after annealing was observed in these samples by TEM [36]. In our case of UFG CP AI, we could not find any experimental evidence of influence of GB segregation on the effect of hardening by annealing. In particular, the specific resistivity of GBs decreases after annealing [23,36], which would not occur in the case of the formation of $G B$ segregation or an increase in its level during annealing. Moreover, the reproducibility of the increase of $\sigma_{0.2}$ after annealing and the decrease of $\sigma_{0.2}$ after subsequent deformation to the level, which is typical for the state before annealing, also excludes any dominant role of segregation in these phenomena. The fact that the effect of hardening by annealing is observed for quite pure Al 99.99 (wt.\%) [21] also supports this conclusion.

On the basis of experimental results and literature data discussed above we can make the following conclusions and suggest some ideas about underlying mechanisms of the observed deformation phenomena: (i) In the HPT-processed CP Al, the relatively small densities of dislocations inside the grains and low-angle GBs certify that strengthening mechanisms caused by these elements of defect structure cannot play any noticeable role in the general strengthening of the HPT-processed UFG AI. In contrast, the strengthening mechanisms caused by the non-equilibrium HAGBs, exhibiting high level of residual strains, should determine the general strengthening in our case. The major micromechanisms of plasticity seem to be motion (glide and climb) of extrinsic GBDs [43,44], emission of lattice dislocations (LDs) from HAGBs $[15,16,45,46]$, glide of the LDs across grains [15,16], accumulation of LDs at the opposite HAGBs [15,16], acceptance of LDs by HAGBs $[13,15,16]$ and transformation of the LDs into GBDs [16]. Diminishing the grain size in these circumstances should decrease the number of extrinsic GBDs which are capable to glide within their HAGBs, form pile-ups at their triple junctions and emit LDs from them. As a result, the emission of LDs should need a higher level of external stress, and a smaller number of LDs should be emitted, which should provide an increase in the strength and a decrease in the ductility of the HPT-processed UFG AI.

(ii) Annealing of the HPT-processed UFG Al at $150{ }^{\circ} \mathrm{C}$ during $1 \mathrm{~h}$ strongly increases its strength and drastically decreases its ductility. The density of dislocations decreases, while the average grain size does not change significantly, and the fraction of HAGBs remains practically the same, however their structure becomes closer to the equilibrium one. In terms of the aforementioned micromechanisms of plasticity, we can suggest that the hardening by annealing is explained by a strong decrease in the number of extrinsic GBDs through their partial annihilation, a significant release of residual elastic strains in the vicinity of HAGBs and, possibly, by pinning of GBDs by impurities migrating along the HAGBs. As a result of these processes, the pile-ups of GBDs at HAGB triple junctions should contain much less GBDs and, therefore, should need much higher external stress to emit LDs into the adjacent grains. This seems to be the main reason for the hardening by annealing in our case. The diminished ductility seems to be mainly related with a decrease in the number of emitted LDs.

(iii) Small additional room-temperature HPT deformation of thus annealed UFG AI decreases the strength (practically to the same characteristic values which were shown by the HPT-processed UFG Al before the annealing), but greatly increases its 
ductility. The crystal lattice microdistorsion level and dislocation densities increase to about the same values as before the annealing. The obtained softening by deformation may be explained in the same terms as before. Indeed, new portion of HPT deformation restores the non-equilibrium state of HAGBs as it follows from even enhanced level of elastic strains. Therefore, the number of GBDs, which are capable to form pile-ups, strongly increases again, the powerful GBD pile-ups form and start to emit LDs under a lower external stress, which can be treated as softening. On the other hand, the number of emitted LDs increases as well, which provides a great enhancement of ductility.

In the following Section 4, we suggest two theoretical models which describe points (ii) and (iii) in detail.

\section{THEORETICAL MODELING}

Based on the experimentally identified key microstructural parameters for the observed effects of annealing-induced hardening and softening by deformation, we propose the following microscopic models which explain these effects and describe the plastic deformation micromechanisms responsible for the unique combination of high strength and functional plasticity in UFG aluminum.

\subsection{Models for transformation of GBD structure in UFG materials}

Consider a two-dimensional model of UFG material with an average grain size $d$. A typical grain of such a material is modeled by a regular hexagon (Figs. 6 and 7). We assume that in the initial state, there are two pile-ups of GBDs of the same lengths and opposite signs, which are pressed by the external shear stress $\tau$ against the GB triple junctions $A$ and $B$ (Figs. 6a and 7a). In the model, for the sake of simplicity, the two GBD pile-ups are modeled by two superdislocations $A$ and $B$ with Burgers vectors $\mathbf{B}$ and $-\mathbf{B}$ respectively (hereinafter we will call them $\pm B$-superdislocations) in the case of UFG material subjected to annealing only (Fig. 6a), and two superdislocations $A$ and $B$ with Burgers vectors $B^{\prime}$ and $-\mathbf{B}^{\prime}$ respectively (hereinafter we will call them $\pm B$ '-superdislocations) in the case of UFG material subjected to annealing and additional HPT (Fig. 7a). The magnitudes of the superdislocation Burgers vectors are $B=n_{\mathrm{pu}} b_{\mathrm{gb}}$ and $B^{\prime}=n_{\mathrm{pu}}^{\prime} b_{\mathrm{gb}}$, where $n_{\mathrm{pu}}$ and $n_{\mathrm{pu}}^{\prime}$ are the numbers of GBDs in the pile-ups, and $b_{\mathrm{gb}}$ is the magnitude of the Burgers vector of a GBD.
In the case of UFG material subjected to annealing only, with no additional HPT, the GBD pileups are characterized by a smaller Burgers vector magnitude, $B=n_{\mathrm{pu}} b_{\mathrm{gb}}, B<B^{\prime}$ (Fig. 6). It should be noted that the length of the pile-ups (which are modeled through $\pm B$-superdislocations) in the case of UFG material subjected to annealing only (Fig. 6) is less than the length of the pile-ups (which are modeled through $\pm B^{\prime}$-superdislocations) in the case of UFG material subjected to annealing with additional HPT (Fig. 7). The pile-up lengths in these two cases are determined by numbers $n_{\mathrm{pu}}$ and $n_{\mathrm{pu}}$ of GBDs, respectively, from which it follows that $n_{\mathrm{pu}}<$ $n_{\text {pu }}^{\prime}$. This is caused by the fact that there are no active sources of GBDs which could produce new GBDs in the case of UFG material subjected to annealing only (see Fig. 6a). On the other hand, in the case of UFG material subjected to annealing with additional HPT, there are active sources $O$ and $O^{\prime}$ of GBDs which fill the pile-ups with the new GBDs in such a way that the pile-up strength (that is the number of GBDs and the pile-up length) is maintained constant (the mechanism of GBD emission from the sources is out of scope of our consideration here). Therefore, the Burgers vector magnitude $B$ ' remains constant.

Let us consider the process of possible transformation of GBD pile-ups in GB triple junctions $A$ and $B$ in the case of external loading of UFG material subjected to annealing only (Fig. 6). Under the combined action of an external shear stress $\tau$ and the stress fields of the superdislocations $A$ and $B$, head GB dislocations of pile-ups split into sessile GB dislocations specified by Burgers vectors $\pm \mathbf{b}_{\mathrm{s} 1}=\mathbf{=} \mathbf{b}+\mathbf{b}_{\mathrm{gb}}$ and mobile lattice dislocations (LDs) with Burgers vectors $\pm \mathbf{b}$ which are emitted from the GB triple junctions $A$ and $B$ into adjacent grain interior (Fig. 6b). In Fig. 6b, these LDs are denoted by number 1 . As a result, the GBD pile-up loses its head $G B$ and its Burgers vector magnitude decreases by $b_{g b}$ as $B-b_{g b}=B_{1}$.

It is worth noting that although the mechanism of emission of perfect and partial LDs from GB triple junctions is well known and theoretically described (see, for example, [45-47]), the present description of LD emission caused by the transformation of the GB dislocation structure is absolutely original and, to the best of our knowledge, was not been considered before.

In terms of the continuum approach [48], the emission of a pair of LDs can be represented as formation of two dipoles $A C$ and BD of LDs with Burgers vectors $\pm \mathbf{b}$. In this case, two mobile LDs 

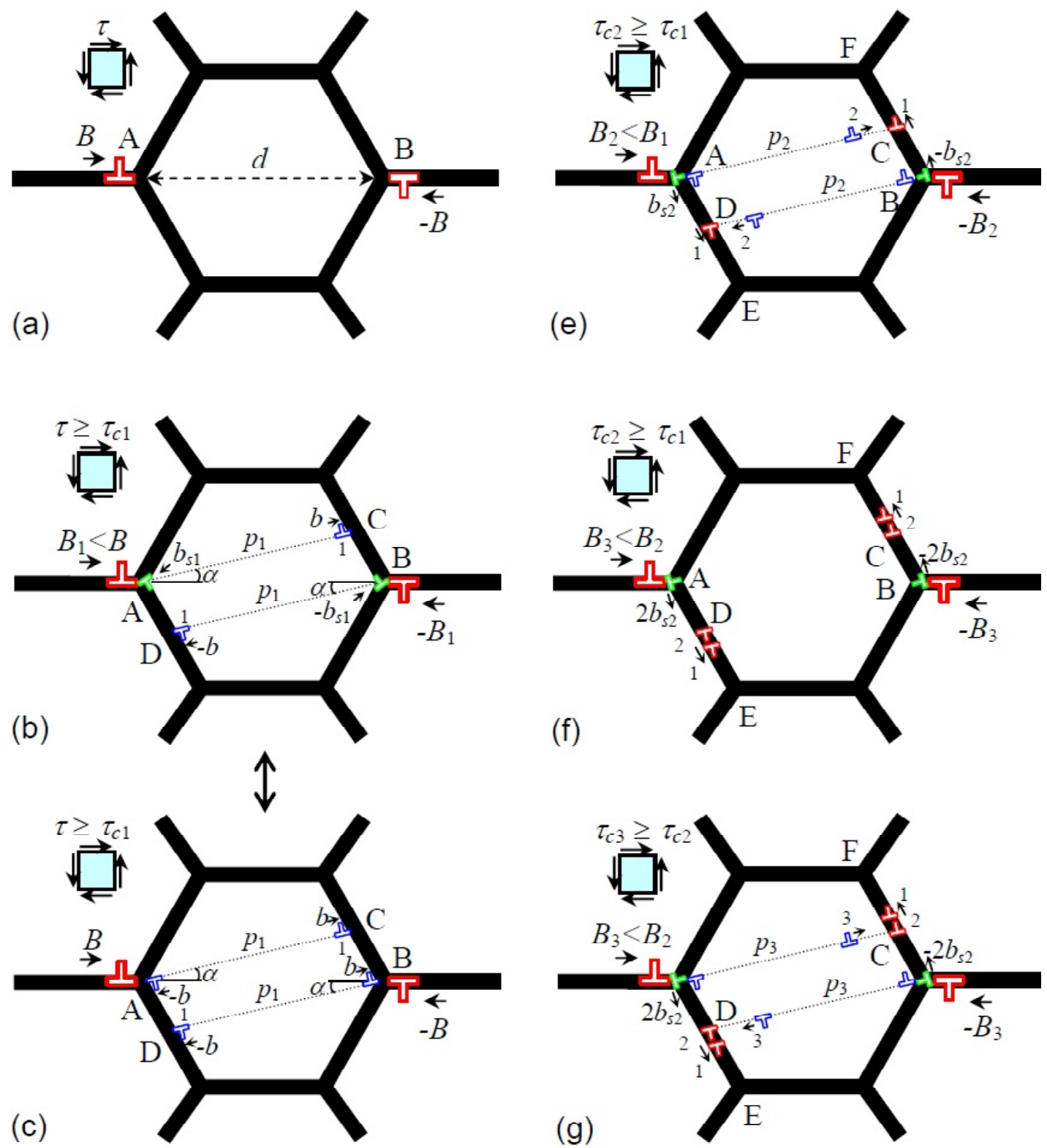

$(f)$
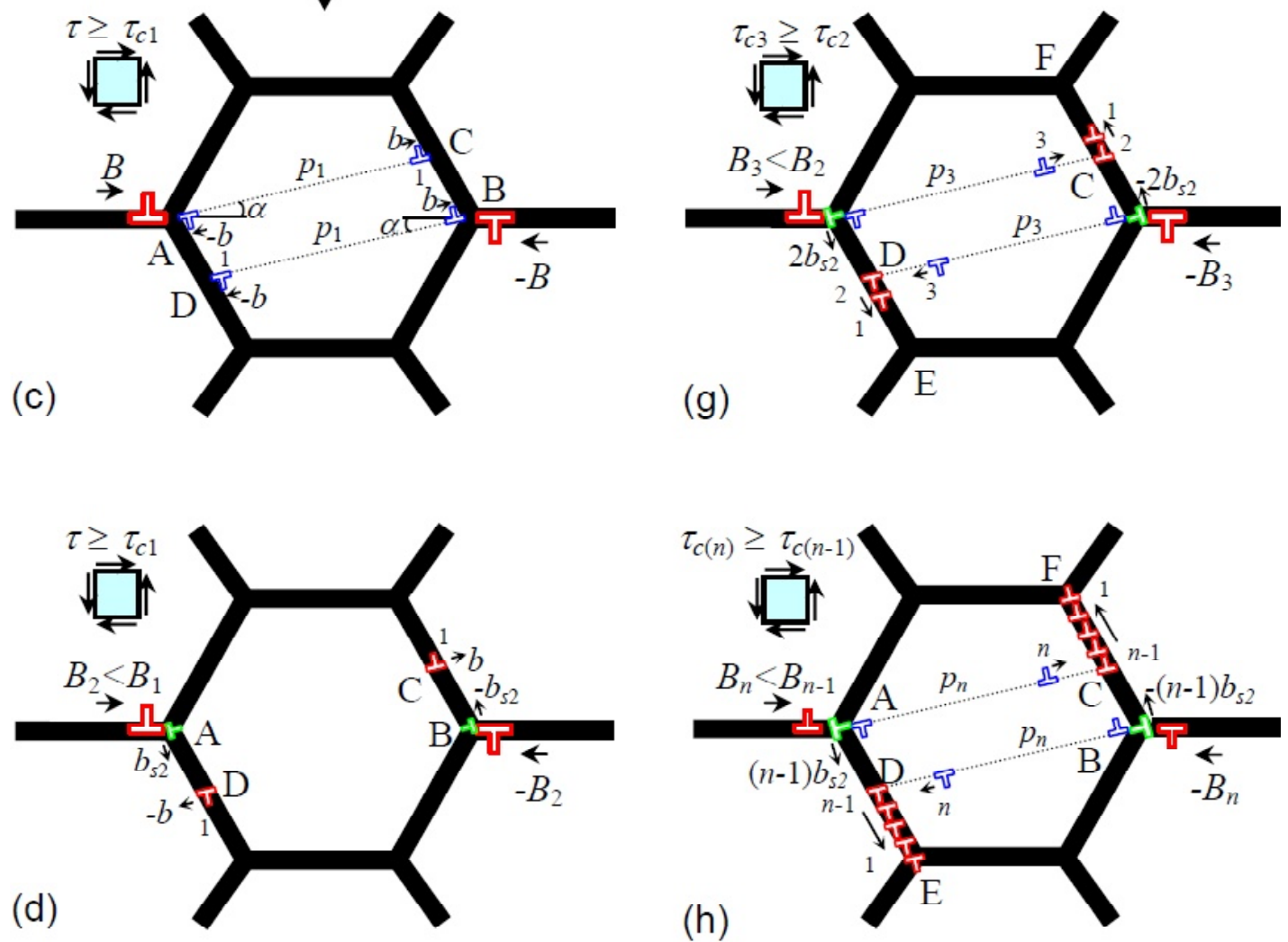

Fig. 6. Model of deformation mechanisms at the scale of a grain in UFG material after annealing only. (a) The initial structure with two pile-ups of GBDs, which are modeled by $\pm B$-superdislocations. $(b, c)$ Emission of the first pair of LDs from triple junctions A and B. (d) Trap of the first pair of LDs by GBs. (e) Emission of the second pair of LDs. (f) Climb of the first pair of LDs along GBs and trap of the second pair of LDs by GBs. (g) Emission of the third pair of LDs. (h) Defect configuration after $n$-th event of LD emission. Red dislocations are GBDs, blue dislocations are LDs, green dislocations are sessile dislocations. 

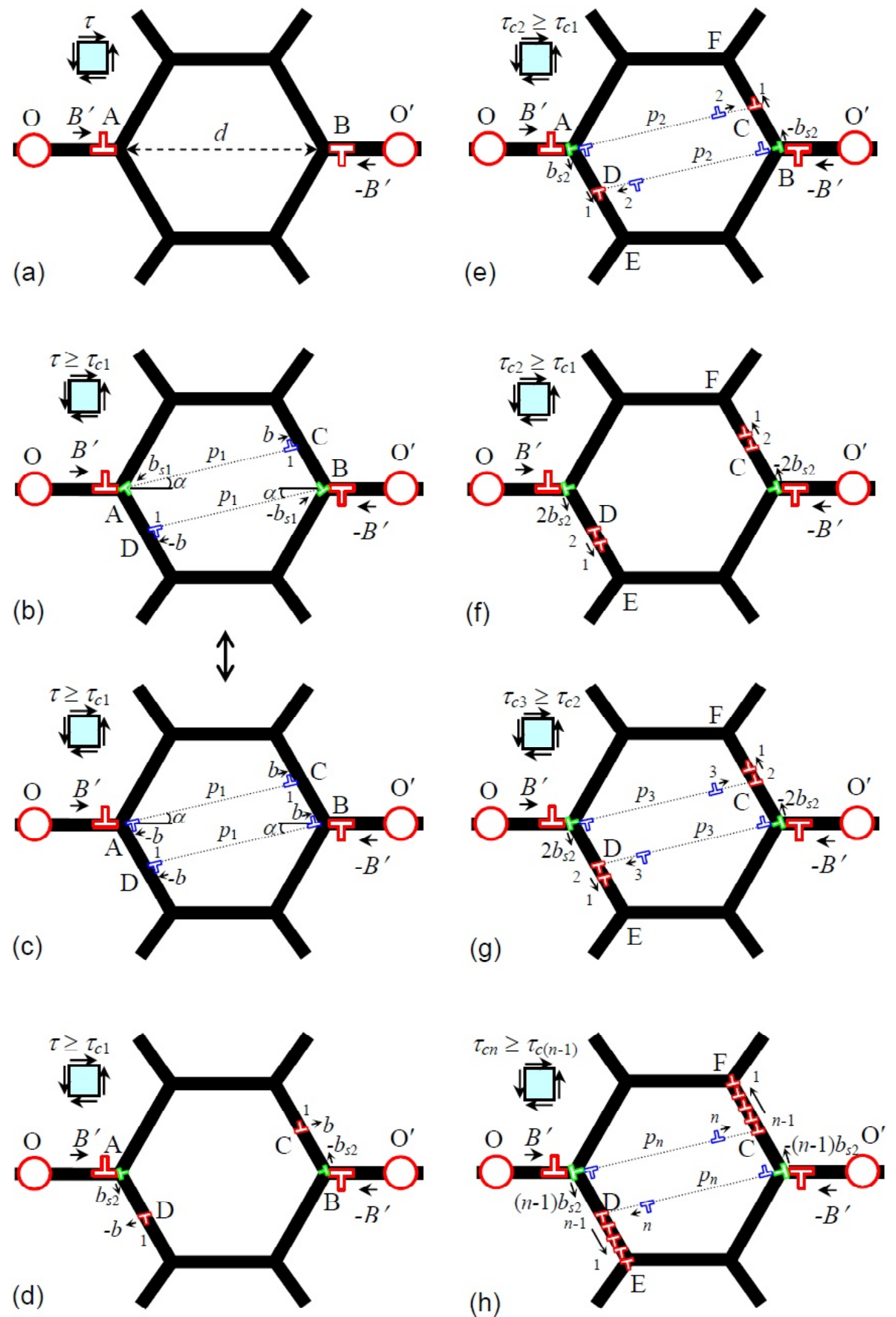

Fig. 7. Model of deformation mechanisms at the scale of a grain in UFG material after annealing and additional HPT. (a) The initial structure with two pile-ups of GBDs, which are modeled by $\pm B$-superdislocations. (b)-(f) Successive emission of LDs from triple junctions $A$ and $B$ and formation of walls CF and DE of climbing GBDs. Red dislocations are GBDs, blue dislocations are LDs, green dislocations are sessile dislocations. 
(a)

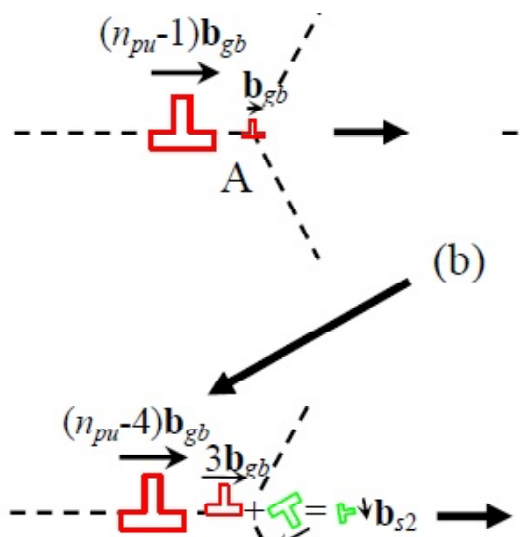

(d)

$$
\mathbf{b}_{s 2}=2 \mathbf{b}_{g b}+\mathbf{b}_{s 1}
$$

(e)

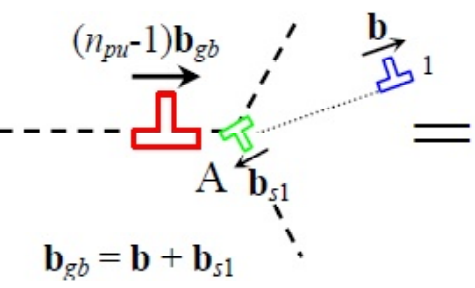

(c)

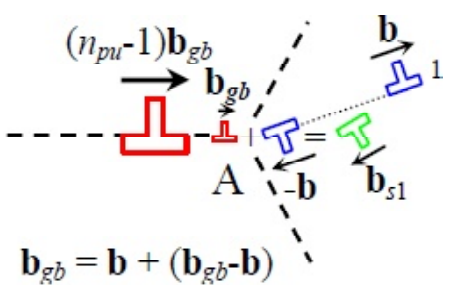

(f)

Fig. 8. Scheme of sessile dislocation formation at the triple junction. (a) The initial defect configuration with a pile-up of GBDs, which is modeled by $\left(n_{\mathrm{pu}}-1\right) b_{\mathrm{gb}}$-superdislocation and a separate head $b_{\mathrm{gb}}$-dislocation. (b,c) Emission of the first lattice $b$-dislocation and formation of the first sessile $b_{s 1}$-dislocation. (d) Formation of the second sessile $b_{\mathrm{s} 2}$-dislocation. $(\mathrm{e}, \mathrm{f})$ Emission of the second lattice $b$-dislocation and transformation of the sessile dislocation.

(mobile $b$-dislocation $\mathrm{C}$ and - $b$-dislocation $\mathrm{D}$ ) glide to the bulk of the grain, while two immobile LDs (immobile - $b$-dislocation $A$ and $b$-dislocation $\mathrm{B}$ ) remain at the triple junctions $A$ and $B$ and overlap with the head GBDs in the GBD pile-ups, in which case the total Burgers vectors $\pm B$ conserve (Fig. $6 \mathrm{c}$ ). The angle $\alpha$ defines the orientation of the LD slip planes. For simplicity, we consider the symmetric case: lattice $b$-dislocation $\mathrm{C}$ and $-b$-dislocation $\mathrm{D}$ are emitted at the same angle $\alpha$ and move over the same distance $p_{1}$ in the grain interior, stopping at the opposite GBs (Fig. 6b,c).

Within the model, the emission of the first pair of LDs becomes possible when the external shear stress $\tau$ reaches a certain critical value $\tau_{c 1}$. At $\tau \geq$ $\tau_{c 1}$, lattice $\pm b$-dislocations constituting the first pair are emitted from the triple junctions $A$ and $B$ and reach the opposite GBs where they are trapped by these GBs and transform into 'new' GBDs which can climb along these GBs (Fig. 6d). At the same time, the sessile $\pm b_{\mathrm{s} 1}$-dislocations react with the new head GBDs of GBD pile-ups forming new sessile dislocations with Burgers vectors $\mathbf{b}_{\mathrm{s} 2}$ (Fig. 6d) and thereby reducing the Burgers vector magnitude of the pile-ups as $B_{1}-b_{g b}=B_{2}$.

Then, the elementary act of the splitting of the head GBDs of pile-ups and the emission of the second pair of lattice $\pm b$-dislocations from the GB triple junctions $A$ and $B$ occurs. As before, the emission of the second pair LDs is modeled by formation of two dipoles $\mathrm{AC}$ and $\mathrm{BD}$ of lattice $\pm b$-dislocations (Fig. 6e). This pair of LDs (denoted by number 2) glides along the same slip planes in the grain as the first pair of LDs (Fig. 6e). On the other hand, the first emitted LDs (those numbered by 1 ) create their stress fields which hamper the emission of new LDs. As a corollary, the second pair of $\pm b$-dislocations may be emitted only if the external shear stress $\tau$ increases up to a new critical value $\tau_{\mathrm{c} 2}>\tau_{\mathrm{c} 1}$. After emission, the second pair $\pm b$-dislocations occupy certain equilibrium positions $p_{2}$ (Fig. 6e) inside the grain, since repulsive forces operate between the first and second pairs of $\pm b$-dislocations, which do not allow the LDs of the second pair to come closely to the LDs of the first pair.

Further, the sessile $\pm b_{\mathrm{s} 2}$-dislocations react with new sessile dislocations (which are formed as a result of the reaction of the new head GBDs with the sessile $b_{s 1}$-dislocations, see Fig. 8 for more details), thus forming new sessile dislocations with Burgers vectors $\pm \mathbf{b}_{\mathrm{s} 3}= \pm 2 \mathbf{b}_{\mathrm{s} 2}$ (Fig. 6f). It is assumed that the first $\pm b$-dislocations climb away from points $C$ and $\mathrm{D}$ allowing the second emitted $\pm b$-dislocations to reach the GBs and facilitating the trap of the second pair of LDs by the GBs AE and BF (Fig. $6 f)$. After that, the emission of the third pair of lattice $\pm b$-dislocations from the triple junctions $\mathrm{A}$ and $\mathrm{B}$ occurs (Fig. $6 \mathrm{~g}$ ). The emission of third $\pm b$-dislocations becomes possible when the external shear stress reaches a critical value $\tau_{\mathrm{c} 3} \geq \tau_{\mathrm{c} 2}$. The third LDs stop in the grain at their stable equilibrium positions at a distance $p_{3}$ from the triple junctions $A$ and B (Fig. 6g). 
In the further emission of pairs of $\pm b$-dislocations, all repeats. Previously emitted LDs are trapped by the GBs $A E$ and BF and transform into new GBDs which can climb along these GBs. In this case, each new emission of LDs requires an increase in the external shear stress $\tau$ to a new critical value $\tau_{c(n)} \geq$ $\tau_{c(n-1)}$ (Fig. 6h). Two walls DE and CF of climbing GBDs are formed due to successive processes of emission of LDs and their trapping by GBs AE and BF (Fig. 6h).

Fig. 8 shows a detailed scheme of sessile dislocation formation and evolution at the GB triple junction A. As is said above, the splitting of the head GBD of the 'left' GBD pile-up (this head GBD is shown separately of the other GBDs composing the $B$-superdislocation in Fig. 8) leads to the emission of the first LD into the grain interior and the formation of the first sessile $b_{s 1}$-dislocation (Figs. 8a and $8 b)$. We have also already mentioned that such a defect configuration is equivalent to the appearance of a dipole of LDs (Fig. 8c). Further, it is easy to see from Fig. $8 \mathrm{c}$ that the Burgers vector magnitude of the $b_{s 1}$-dislocation is roughly three times larger than that of the GBD and, therefore, the first two head GBDs of the pile-up react with the $b_{s 1}$-dislocation with no subsequent emission of LDs from the triple junction. Only the third head GBD of the pile-up, after such a reaction, is capable to emit a new LD into the grain interior (Fig. 8d). Thus, the sessile $b_{\mathrm{s} 1}$-dislocation reacts with three head $b_{\mathrm{gb}}$-dislocations of the GBD pile-up, forming a new sessile dislocation with Burgers vector $\mathbf{b}_{\mathrm{s} 2}$ (Fig. 8d). After that, the next head GBD comes to the triple junction (Fig. $8 e$ ) and splits into a lattice $b$-dislocation (denoted by number 2), which is emitted from the triple junction into the grain interior, and a new sessile dislocation with Burgers vector $\mathbf{b}_{\mathrm{s} 1}$ (Fig. 8f). Then, the reaction process of next three head GBDs with the new sessile $b_{\mathrm{s} 1}$-dislocation repeats (see Fig. 8d). As a result, a new sessile $b_{\mathrm{s} 2}$-dislocation is formed which combines with the previously formed sessile $b_{\mathrm{s} 2}$-dislocation into a sum sessile dislocation with Burgers vector $\mathbf{b}_{\mathrm{s} 3}=2 \mathbf{b}_{\mathrm{s} 2}$. This process of sessile dislocation transformations can repeat many times, leading to a gradual increase in the magnitude of the sessile dislocation Burgers vector $\mathbf{b}_{\mathrm{s} n}=(n-1) \mathbf{b}_{\mathrm{s} 2}$.

As a result, we can expect a combination of deformation strengthening, which is caused by elastic repulsion of gliding LDs from the walls of climbing GBDs, with rather high ductility of the system, provided by the generation and glide of GBDs forming the pile-ups, the emission and glide of LDs across the grain to the opposite GBs, and the climb of new GBDs, transformed from the trapped LDs, along these GBs.

In the case of UFG material subjected to annealing and additional HPT, the process of emission of LDs from GB triple junctions $A$ and $B$ and the formation of walls of climbing GBDs occurs in a similar scenario (Fig. 7). However, in this case, the strength of GBD pile-ups (that is the magnitude $B^{\prime}$ of their sum Burgers vector) is maintained constant as the pile-ups are fueled by new GBDs from their GB sources $O$ and $O^{\prime}$ (Fig. 7) which leads to a significant decrease in the critical stresses $\tau_{c n}^{\prime}<\tau_{c n}$ required to emit new LDs. As a result, the emission of LDs stops much later than in the case when the strength of GBD pile-ups decreases in parallel with each emission of LDs (Fig. 6). Therefore, we can expect good ductility in the second case, which is explained by the existence of GB sources for generation of GBDs capable to glide along the GBs and hence by the fast fueling of the GBD pile-ups. The key point here is the lasting operation of GBD sources $O$ and $O$ ' which are assumed to be formed during additional HPT of the sample. The source activity provides the stable work of the chain of plasticity mechanisms: GBD glide - LD glide - GBD climb.

Thus, as follows from the above models (Figs. 6 and 7), UFG materials subjected to annealing only (Fig. 6) should exhibit relatively higher strength and lower ductility than UFG materials subjected to annealing and additional HPT (Fig. 7) that is in a good accordance with our experimental results (see Section 3).

Let us consider now the energy characteristics of emission of the lattice $\pm b$-dislocations from the GB triple junctions $A$ and $B$ in the two cases, for UFG materials subjected to annealing only (Fig. 6) and for UFG materials subjected to annealing and additional HPT (Fig. 7).

\subsection{Energetic characteristics of transformation of GBD structure in UFG materials}

To analyze the models suggested in the previous Section, we used a semi-analytical energetic approach which is based on calculation of the total energy of the defect structure under consideration per unit length of parallel dislocations composing this defect structure. On every key step of the structure transformation, we calculated the difference between the total energies characterizing the structure after and before the transformation event, and analyzed the energy differences numerically with 
determining the critical conditions for these transformations. A similar approach was used before many times [43-47,49-53], so in the present paper, we do not describe it in detail and address the reader to the earlier works.

Consider the conditions which are necessary for the energetically favorable emission of any pair of lattice $\pm b$-dislocations from the GB triple junctions A and B (Fig. $6 \mathrm{~h}$ ). In doing so, we study the defect system transformation from the $(n-1)$-th state with a total energy $W_{n-1}$, corresponding to the state of the system with ( $n$-1) pairs of $\pm b$-dislocations, which is formed just before the moment of emission of the $n$-th pair of $\pm b$-dislocations, to the $n$-th state with a total energy $W_{n}$, corresponding to the emission of the $n$-th pair of $\pm b$-dislocations. Such a transformation of the defect system is energetically favorable if $\Delta W_{n}=W_{n}$ $W_{n-1}<0$.

The energy difference $\Delta W_{n}$ is determined by the expression:

$$
\Delta W_{n}=E_{s(n)}^{2 b}+E_{\mathrm{int}(n)}^{2 b-2 b}+E_{\mathrm{int}(n)}^{B-2 b}+E_{\mathrm{int}(n)}^{2 b-b}+E_{\mathrm{int}(n)}^{b s-2 b}+E_{\tau(n)},
$$

where $E_{s(n)}^{2 b}$ is the self energy of dipole $\mathrm{AC}(\mathrm{BD})$ of $\pm b$-dislocations in the $n$-th state of the defect system; $E_{\text {int }(n)}^{2 b-2 b}$ is the energy of elastic interaction between dipoles AC and BD of $\pm b$-dislocations; $E_{\mathrm{int}(n)}^{B-2 b}$ is the energy of elastic interaction of dipole $\mathrm{AB}$ of $\pm B_{n}$-superdislocations with dipoles $\mathrm{AC}$ and $\mathrm{BD}$ of $\pm b$-dislocations; $E_{\mathrm{int}(n)}^{2 b-b}$ is the sum of the energies specifying the elastic interaction of dipoles AC and BD of $\pm b$ dislocations and walls DE and CF of climbing $\pm b$-dislocations; $E_{\mathrm{int}(n)}^{\mathrm{bs}-2 b}$ is the energy of elastic interaction of dipoles $\mathrm{AC}$ and $\mathrm{BD}$ of the lattice $\pm b$-dislocations with the sessile $\pm(n-1) b_{\mathrm{s} 2}$-dislocations; $E_{\tau(n)}$ is the sum of interaction energies of the applied shear stress $\tau$ with dipoles $A C$ and $B D$ of $\pm b$-dislocations and walls $D E$ and $\mathrm{CF}$ of climbing $\pm b$-dislocations.

The self energy $E_{s(n)}^{2 b}$ of dipole $\mathrm{AC}(\mathrm{BD})$ of $\pm b$-dislocations is given by standard formulas [50]:

$$
E_{s(n)}^{2 b}=2 D b^{2}\left(\ln \frac{p_{n}-r_{c}}{r_{c}}+1\right),
$$

where $D=G /[2 \pi(1-v)], G$ is the shear modulus, $v$ is the Poisson ratio, $p_{n}$ is the path moved by the $n$-th $\pm b$ dislocations, $r_{c} \approx b$ is the cut-off radius of the stress fields of $\pm b$-dislocations.

To calculate the interaction energies $E_{\mathrm{int}(n)}^{2 b-2 b}, E_{\mathrm{int}(n)}^{B-2 b}, E_{\mathrm{int}(n)}^{2 b-b}$ and $E_{\mathrm{int}(n)}^{b s-2 b}$ between different defects, we used the standard procedure of calculating the work spent to nucleate one defect (or one group of defects) in the stress field of another defect (or another group of defects), see, for example, paper [50] for details. In doing so, we got the final expressions for the interaction energies as follows:

$$
\begin{aligned}
& E_{\mathrm{int(n)}}^{2 b-2 b}=\frac{D b^{2}}{2}\left\{\ln \frac{\left[\left(p_{n}-x_{0}^{\prime}\right)^{2}+y_{0}^{2}\right]\left[\left(p_{n}-x_{0}^{\prime}\right)^{2}+y_{0}^{2}\right]}{\left(x_{0}^{\prime 2}+y_{0}^{2}\right)^{2}}-\frac{4 y_{0}^{2}}{x_{0}^{\prime 2}+y_{0}^{2}}+\frac{2 y_{0}^{2}}{\left(p_{n}-x_{0}^{\prime}\right)^{2}+y_{0}^{2}}+\frac{2 y_{0}^{2}}{\left(p_{n}+x_{0}^{\prime}\right)^{2}+y_{0}^{2}}\right\}, \\
& E_{\mathrm{int}(n)}^{B-2 b}=D B_{n} b\left(\cos \alpha \ln \left[\frac{d^{2}+2 d p_{n} \cos \alpha}{p_{n}^{2}}+1\right]+\frac{2 d p_{n} \sin ^{2} \alpha}{d^{2}+p_{n}^{2}+2 d p_{n} \cos \alpha}\right), \\
& E_{\mathrm{int}(n)}^{2 b-b}=D b^{2} \sum_{i=1}^{n-1}\left(\ln \frac{\left(p_{n}-x_{0}\right)^{2}+y_{i}^{2}}{x_{0}^{2}+y_{i}^{2}}-\frac{2 y_{i}^{2}\left(p_{n}^{2}-2 p_{n} x_{0}\right)}{\left[\left(p_{n}-x_{0}\right)^{2}+y_{i}^{2}\right]\left(x_{0}^{2}+y_{i}^{2}\right)}\right), \\
& E_{\mathrm{int}(n)}^{s-2 b}=D b b_{s n}\left\{\cos (\alpha+\beta)\left(\ln \frac{x_{0}^{\prime \prime 2}+y_{0}^{\prime \prime 2}+p_{n}^{2}-2 p_{n} x_{0}^{\prime \prime} \cos (\alpha+\beta)-2 p_{n} y_{0}^{\prime \prime} \sin (\alpha+\beta)}{x_{0}^{\prime \prime 2}+y_{0}^{\prime \prime 2}}-\ln \frac{p_{n}^{2}}{b^{2}}\right)+\right. \\
& \left.\frac{2 y_{0}^{\prime \prime}\left[x_{0}^{\prime \prime} \sin (\alpha+\beta)-y_{0}^{\prime \prime} \cos (\alpha+\beta)\right]}{x_{0}^{\prime \prime 2}+y_{0}^{\prime \prime 2}}-\frac{2\left[y_{0}^{\prime \prime}-p_{n} \sin (\alpha+\beta)\right]\left[x_{0}^{\prime \prime} \sin (\alpha+\beta)-y_{0}^{\prime \prime} \cos (\alpha+\beta)\right]}{x_{0}^{\prime \prime 2}+y_{0}^{\prime \prime 2}+p_{n}^{2}-2 p_{n} x_{0}^{\prime \prime} \cos (\alpha+\beta)-2 p_{n} y_{0}^{\prime \prime} \sin (\alpha+\beta)}\right\},
\end{aligned}
$$

where $x_{0}=p_{1}, x_{0}^{\prime}=p_{1}-p_{n}, x_{0}^{\prime \prime}=d \cos \beta, y_{0}=h, y_{0}^{\prime \prime}=d \sin \beta, x_{i}=(i-1) \operatorname{acot}\left(60^{\circ}+\alpha\right), y_{i}=(i-1) a, p_{1}=d \sqrt{3} /\left[2 \sin \left(60^{\circ}+\alpha\right)\right]$, $h=d \sin \alpha / \cos \left(\alpha-30^{\circ}\right), B_{n}=\left(n_{\mathrm{pu}}-3 n+2\right) b_{\mathrm{gb}}, b_{\mathrm{sn}}=(n-1) b_{\mathrm{s} 2}, b_{\mathrm{s1}}=\sqrt{b_{g b}^{2}+b^{2}-2 b_{g b} b \cos \alpha}, b_{s 2}=\sqrt{4 b_{g b}^{2}+b_{s 1}^{2}-4 b_{g b} b_{s 1} \cos \alpha}$, $\beta=\arcsin \left(b_{\mathrm{s} 1} / b_{\mathrm{s} 2} \sin \alpha\right), n$ is the number of emitted lattice $\pm b$-dislocations, $a$ is the lattice parameter. 


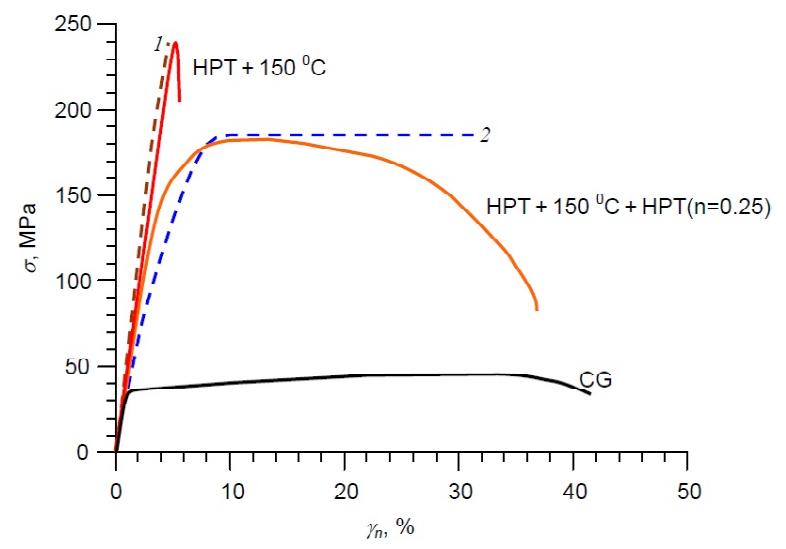

Fig. 9. Theoretical dependences of the flow stress $\sigma$ on the value of plastic deformation $\gamma_{n}$ (dashed curves) in comparison with the experimental data (solid curves).

The total interaction energy of the external shear stress $\tau$ with dipoles $\mathrm{AC}$ and $\mathrm{BD}$ of $\pm b$-dislocations and walls $\mathrm{DE}$ and $\mathrm{CF}$ of climbing $\pm b$-dislocations reads:

$E_{\tau(n)}=-b \tau \cos 2 \alpha\left[p_{n}+2(n-1) a\right]$.

In a similar way, the energy difference $\Delta W_{n}^{\prime}$, which corresponds to the defect structure transformation shown in Fig. 7 , is given by

$$
\Delta W_{n}^{\prime}=\Delta W_{n}\left(B_{n} \rightarrow B^{\prime}\right) .
$$

Using formulas (3)-(10), we calculated the energy differences $\Delta W_{n}$ and $\Delta W_{n}^{\prime}$. The stable equilibrium positions $p_{n}$ of the previously emitted $\pm b$-dislocations correspond to minima in the $\Delta W_{n}\left(p_{n}\right)$ and $\Delta W_{n}^{\prime}\left(p_{n}\right)$ dependences and can be found from the equations $\Delta W_{n} / \partial p_{n}=0$ and $\Delta W_{n}^{\prime} / \partial p_{n}=0$, respectively. With these results on hand, we also calculated the critical shear stress $\tau_{c(n)}$ that is the minimum stress at which the emission of the $n$-th pair of LDs is energetically favorable. The critical stress $\tau_{c(n)}$ can be found from the conditions that $\Delta W_{n}\left(p=p^{\prime}\right)=0\left(\right.$ where $\left.p^{\prime}=1 \mathrm{~nm}\right)$,

$$
\left.\Delta W_{n}\right|_{p_{n}>p^{\prime}}<0 \text { and }\left.\frac{\partial \Delta W_{n}}{\partial p_{n}}\right|_{p_{n}>p^{\prime}} \leq 0 \text {. }
$$

The critical stress $\tau_{c(n)}$ is related to the flow stress $\sigma$ as follows: $\sigma=2 \tau_{c(n)} / \cos 2 \alpha$.

Calculation of the critical stress $\tau_{\mathrm{c}(n)}^{\prime}$ corresponding to the emission of LDs in situation shown in Fig. 7 is similar to the calculation of the critical stress $\tau_{c(n)}$.

Based on the aforementioned calculations of critical stresses $\tau_{\mathrm{c}(n)}$ and $\tau_{\mathrm{c}(n)}^{\prime}$, we also calculated the dependence of the flow stress $\sigma$ on the value of plastic shear deformation $\gamma_{n}$. The latter can be estimated by the following expression: $\gamma_{n} \approx 2 n b / d$. To calculate the dependences $\sigma\left(\gamma_{n}\right)$ for UFG aluminum, we used the following values of material parameters: $G=27 \mathrm{GPa}, v=0.31, a=0.405 \mathrm{~nm}, b=a \sqrt{2} / 2$, $b_{\mathrm{gb}} \approx 0.1 \mathrm{~nm}$ [48]. Other parameters of the defect system were taken as $d=1000 \mathrm{~nm}$ and $\alpha=20^{\circ}$.

The dependences $\sigma\left(\gamma_{n}\right)$ calculated numerically (Fig. 9) for UFG aluminum subjected to annealing, in which case we took $n_{\mathrm{pu}}=15$ (dashed curve 1), and to annealing and additional HPT, in which case we took $n_{\mathrm{pu}}^{\prime}=30$ (dashed curve 2). For comparison, our experimental curves given by solid lines are also shown in Fig. 9. As is seen, the theoretical results are in a good agreement with the experimental data.

It is worth noting that our theoretical results also well correspond with those of computer simulations by Hasnaoui et al. [41] who reported on a reduction of plastic strain (ductility) when GBs and triple junction regions approach more equilibrium conditions in nanocrystalline Ni with mean grain size of $12 \mathrm{~nm}$. Although it seems evident that underlying mechanisms of plasticity in that case of extremely fine nanograins should be rather different as compared with our case, the general issue is the same: the closer GB structures are to their equilibrium states, the lower ductility of the UFG (or nanocrystalline) material should be expected.

\section{SUMMARY AND CONCLUSIONS}

For the first time, we have demonstrated experimentally the hardening by annealing in a temperature range of $90-200^{\circ} \mathrm{C}$ for HPT-processed CP Al and the implementation of its high ductility at the ductility level of CG Al, while maintaining high strength of the HPT-processed sample, by repeating the low temperature annealing followed by a slight additional deformation by HPT. We have shown that the UFG structure of the HPT-processed samples is characterized by the following specific features: (i) the most of GBs are non-equilibrium HAGBs, (ii) the initial dislocation density was rather low $\left(4.110^{12} \mathrm{~m}^{-2}\right)$ and mostly attributed to GBs, (iii) the grain interior was nearly free from dislocations. The annealing leads to decrease of crystal lattice distorsions near HAGBs and 3 times decrease of dislocation density without change in $G B$ distribution on misorientation angle and without significant change in grain size. The additional small deformation almost restored the dislocation density to the value which is typical for HPT-processed samples. 
Based on the obtained experimental results, we have suggested two theoretical models which explain the hardening by annealing and the implementation of high plasticity while maintaining a high level of strength in UFG structures. These theoretical models describe the main micromechanisms of plastic deformation in UFG aluminum subjected to these treatments. In particular, the plastic deformation is assumed to be realized through the emission of LDs from triple junctions of GBs containing pile-ups of GBDs, the glide of these LDs across neighboring grains, their accumulation at and climb along the opposite GBs. The energy characteristics and the critical stresses of LD emission have been determined in the two different cases, for UFG aluminum subjected to annealing only and to annealing with small additional HPT deformation. In the models, these two regimes differ from each other by the assumptions that, in the first case of annealing with no additional HPT deformation, there are no active GBD sources which are capable to generate many GBDs and to support their constant number in the GBD pile-up, while in the second case of annealing with small additional HPT deformation, such active GBD sources do exist. As a result, in the first case, the GBD pile-ups are relatively weak and can emit a limited number of LDs under a relatively higher external stress, which results in the mechanical behavior characterized by very high strength and low ductility. In contrast, in the second case, the GBD pile-ups are relatively strong and can emit a large number of LDs under a relatively lower external stress, which results in a relatively lower strength and higher ductility of the UFG material. The resulting theoretical dependences of the flow stress on the plastic deformation value have been calculated which demonstrate good qualitative and quantitative agreement with our experimental data.

\section{ACKNOWLEDGEMENTS}

X-ray diffraction studies were provided using the equipment of the Resource Centre for X-ray Diffraction Methods of Research of Saint Petersburg State University. EBSD analyses were conducted on the equipment of the Interdisciplinary Resource Centre for Nanotechnology of Saint Petersburg State University.

\section{REFERENCES}

[1] Z. Horita, T. Fujinami, M. Nemoto and T.G. Langdon // Met. Mater. Trans. A 31 (2000) 691.

[2] R.Z. Valiev, R.K. Islamgaliev and I.V. Alexandrov // Prog. Mater. Sci. 45 (2000) 103.
[3] J.K. Kim, H.K. Kim, J.W. Park and W.J. Kim // Scr. Mater. 53 (2005) 1207.

[4] A. Azushima, R. Kopp, A. Korhonen, D.Y. Yang, F. Micari, G.D. Lahoti, P. Groche, J. Yanagimoto, N. Tsuji, A. Rosochowski and A. Yanagida // CIRP J. Manuf. Sci. Technol. 57 (2008) 716.

[5] A.S. Khan, B. Farrokh and L. Takacs // Mater. Sci. Eng. A 89 (2008) 77.

[6] B. Farrokh and A.S. Khan // Int. J. Plasticity 25 (2009) 715.

[7] A.S. Khan and C.S. Meredith // Int. J. Plasticity 26 (2010) 189.

[8] C.S. Meredith and A.S. Khan // Int. J. Plasticity 30 (2012) 202.

[9] Y. Aoyagi, R. Kobayashi, Y. Kaji and K. Shizawa // Int. J. Plasticity 47 (2013) 13.

[10] J. Liu, A.S. Khan, L. Takacs, C.S. Meredith // Int. J. Plasticity 64 (2015) 151.

[11] C.S. Meredith and A.S. Khan // J. Mater. Process. Tech. 219 (2015) 257.

[12] I. Sabirov, M.R. Barnett, Y. Estrin and P.D. Hodgson // Scr. Mater. 61 (2009) 181.

[13] C.-F. Yang, J.-H. Pan and T.-H. Lee // J. Alloys Compd. 468 (2009) 230.

[14] A.S. Khan and J. Liu // Intern. J. Plasticity 86 (2016) 56.

[15] H. Van Swygenhoven // Mater. Sci. Eng. A 483 (2008) 33.

[16] F. Mompiou, D. Caillard, M. Legros and H. Mughrabi // Acta Mater. 60 (2012) 3402.

[17] X. Sauvage, G. Wilde, S.V. Divinski, Z. Horita and R.Z. Valiev // Mater. Sci. Eng. A 540 (2012) 1.

[18] Y. Aoyagi, T. Tsuru and T. Shimokawa // Int. J. Plasticity 55 (2014) 43.

[19] X. Huang, N. Hansen and N. Tsuji // Science 312 (2006) 249.

[20] J.R. Bowen // Mater. Sci. Eng. A 483 (2008) 231.

[21] N. Kamikawa, X. Huang, N. Tsuji and N. Hansen // Acta Mater. 57 (2009) 4198.

[22] B.N. Semenov, I.V. Smirnov, Yu.V. Sud'enkov and N.V. Tatarinova // Mater. Phys. Mech. 24 (2015) 319.

[23] A.M. Mavlyutov, A.S. Bondarenko, M.Yu. Murashkin, E.V. Boltynjuk, R.Z. Valiev and T.S. Orlova // JALCOM698 (2017) 539.

[24] A.M. Mavlyutov, T.A. Latynina, M.Yu. Murashkin, R.Z. Valiev and T.S. Orlova // Phys. Solid State 59 (2017) 1970.

[25] Y. Miyajima, S.-Y. Komatsu, M. Mitsuhara, S. Hata, H. Nakashima and N. Tsuji // Phil. Mag. 95 (2015) 1139. 
[26] M. Kato // Mater. Sci. Eng. A 516 (2009) 276.

[27] M.A. Abdulstaar, E.A. El-Danaf, N.S. Waluyo and L. Wagner // Mater. Sci. Eng. A 565 (2013) 351.

[28] M.M. Salem, E.A. El-Danaf, A.A. El-Enany and A.A. Radwan // J. Eng. Appl. Sci. 55 (2008) 125.

[29] A.P. Zhilyaev and T.G. Langdon // Prog. Mater. Sci. 53 (2008) 893.

[30] F.J. Humphreys // J. Microscopy 195 (1999) 170.

[31] G.K. Williamson and R.E. Smallman // Phil. Mag. 1 (1956) 34.

[32] H. Miyamoto, K. Ota and T. Mimaki // Scr. Mater. 54 (2006) 1721.

[33] E.A. El-Danaf, M.S. Soliman, A.A. Almajid and M.M. El-Rayes // Mater. Sci. Eng. A 458 (2007) 226.

[34] B. Talebanpour, R. Ebrahimi and K. Janghorban // Mater. Sci. Eng. A 527 (2009) 141.

[35] W. Zeng, Y. Shen, N. Zhang, X. Huang, J. Wang, G. Tang and A. Shan // Scr. Mater. 66 (2012) 147.

[36] T.S. Orlova, A.M. Mavlyutov, A.S. Bondarenko, I.A. Kasatkin, M.Yu. Murashkin and R.Z. Valiev // Phil. Mag. 96 (2016) 2429.

[37] R.K. Islamgaliev and R.Z. Valiev // Phys. Solid State 37 (1995) 1979.

[38] R.K. Islamgaliev and R.Z. Valiev // Phys. Met. Metallogr. 87 (1999) 215.

[39] M. Liu, H.J. Roven, T. Ungár, L. Balogh, M.Y. Murashkin and R.Z. Valiev // Mater. Sci. Forum 584 (2008) 528.
[40] H. Rosner, Ch. Kubel, Yu. Ivanisenko, L. Kurmanaeva, S.V. Divinski, M. Peterlechner and G. Wilde // Acta Mater. 59 (2011) 7380.

[41] A. Hasnaoui, H. Van Swygenhoven and P.M. Derlet // Acta Mater. 50 (2002) 3927.

[42] J. Hu, Y.N. Shi, X. Sauvage, G. Sha and K. Lu // Science 355 (2017) 1292.

[43] M.Yu. Gutkin, I.A. Ovid'ko and N.V. Skiba // Acta Mater. 51 (2003) 4059.

[44] M.Yu. Gutkin, I.A. Ovid'ko and N.V. Skiba // Acta Mater. 52 (2004) 1711.

[45] M.Yu. Gutkin, I.A. Ovid'ko and N.V. Skiba // J. Phys. D: Appl. Phys. 38 (2005) 3921.

[46] I.A. Ovid'ko and N.V. Skiba // Int. J. Plasticity 62 (2014) 50.

[47] I.A. Ovid'ko and N.V. Skiba // Scripta Mater. 71 (2014) 33.

[48] J.P. Hirth and J. Lothe, Theory of Dislocations (Wiley, New York, 1982).

[49] M.Yu. Gutkin, I.A. Ovid'ko and N.V. Skiba // Phys. Rev. B74 (2006) 172107 (4 pages).

[50] M.Yu. Gutkin, I.A. Ovid'ko and N.V. Skiba // Phil. Mag. 88 (2008) 1137.

[51] I.A. Ovid'ko and N.V. Skiba // Rev. Adv. Mater. Sci. 43 (2015) 22.

[52] I.A. Ovid'ko and N.V. Skiba // Rev. Adv. Mater. Sci. 47 (2016) 66.

[53] I.A. Ovid'ko and N.V. Skiba // Rev. Adv. Mater. Sci. 50 (2017) 31. 